41th "Jaszowiec" International School and Conference on the Physics of Semiconductors, Krynica-Zdrój 2012

\title{
Spin Hall Effect in a Two-Dimensional Electron Gas with Constant Dresselhaus and Random Rashba Spin-Orbit Interactions
}

\author{
A. DYRDAE ${ }^{a}$ AND J. BARNAS $\hat{S}^{a, b}$ \\ ${ }^{a}$ Faculty of Physics, Adam Mickiewicz University, Umultowska 85, 61-614 Poznań, Poland \\ ${ }^{b}$ Institute of Molecular Physics, Polish Academy of Sciences, M. Smoluchowskiego 17, 60-179 Poznań, Poland \\ Spin Hall effect in a two-dimensional electron gas with uniform Dresselhaus and random Rashba spin-orbit \\ interactions is considered theoretically. Using Kubo formalism we derive some analytical formula for the spin Hall \\ conductivity. It is shown that the contribution due to randomly fluctuating Rashba field disappears in the limit \\ of strong Dresselhaus coupling.
}

PACS: 73.63.-b, 72.25.Dc, 73.50.Bk

\section{Introduction}

Four decades ago Dyakonov and Perel predicted transverse spin current (or spin accumulation) induced by an electric current flowing through system with spin-orbit interactions $[1,2]$. This effect was reconsidered later by Hirsch [3], and is known now as the spin Hall effect (SHE). Since then, SHE was extensively studied mainly because it allows to manipulate the spin degree of freedom with electric field only. Such a possibility is interesting from fundamental reasons, but it is also very important for further development of spintronic devices, especially those whose functionality is based on magnetic switching and magnetic dynamics.

Generally, SHE may occur in metallic and semiconducting systems with spin-orbit interaction. Furthermore, mechanisms leading to SHE may be either of intrinsic or extrinsic origins (for overview see Refs. $[4-7]$ ). The extrinsic SHE arises from spin-orbit scattering on impurities (side jump and skew scattering). However, there is also intrinsic spin-orbit interaction having crystal lattice periodicity and contributing to the relevant band structure. The corresponding intrinsic SHE is a consequence of an unusual trajectory of the charge carriers in the momentum space.

In this paper we consider SHE in a two-dimensional electron gas (2DEG). The latter can be considered as a basic model of semiconducting structures like quantum wells and heterojunctions. The dominant spin-orbit interactions in 2DEG are the Rashba and the Dresselhaus ones. SHE in 2DEG was extensively studied in recent years. For instance, Sinova et al. [8] reported a universal value of intrinsic spin Hall effect in 2DEG with the Rashba spin-orbit interaction. The effect, however, is totally suppressed when one takes into account the presence of impurities with point-like scattering potentials.

It has been also shown that randomness of spin-orbit interaction (due to random distribution of dopant ions, some imperfections of quantum well interfaces, etc.) can play an important role in spin manipulation and spin transport [9]. For instance, fluctuating Rashba field can induce SHE in 2DEG [10]. However, the corresponding spin Hall conductivity is not universal, but depends on the momentum and spin relaxation times.

\section{Model}

We consider SHE in a 2DEG with constant Dresselhaus spin-orbit coupling and spatially fluctuating Rashba interaction (see Fig. 1). The latter will be treated perturbatively. In the momentum space, Hamiltonian of the system takes the following form (we use the units with $\hbar=1)$ :

$$
H=\sum_{k k^{\prime}} \Psi_{k}^{\dagger} H_{k k^{\prime}} \Psi_{\boldsymbol{k}^{\prime}}
$$

with

$$
\begin{array}{r}
H_{\boldsymbol{k} \boldsymbol{k}^{\prime}}=\frac{k^{2}}{2 m} \delta_{\boldsymbol{k} \boldsymbol{k}^{\prime}}+\beta\left(\sigma_{x} k_{x}-\sigma_{y} k_{y}\right) \delta_{\boldsymbol{k} \boldsymbol{k}^{\prime}} \\
+\frac{\lambda_{\boldsymbol{k} \boldsymbol{k}^{\prime}}}{2}\left[\sigma_{x}\left(k_{y}+k_{y}^{\prime}\right)-\sigma_{y}\left(k_{x}+k_{x}^{\prime}\right)\right] .
\end{array}
$$

Here $m$ is the electron effective mass, $\beta$ is the Dresselhaus spin-orbit constant, $\lambda_{\boldsymbol{k} \boldsymbol{k}^{\prime}}$ describe the random Rashba spin-orbit interaction, and $\sigma_{\alpha}$ (for $\alpha=x, y, z$ ) are the Pauli matrices. We assume that the spatially averaged Rashba interaction vanishes $(\langle\lambda(\boldsymbol{r})\rangle=0)$, but there is a nonzero correlation function $\left(\left\langle\lambda(\boldsymbol{r}) \lambda\left(\boldsymbol{r}^{\prime}\right)\right\rangle=C\left(\boldsymbol{r}-\boldsymbol{r}^{\prime}\right)\right)$. Furthermore, we assume this correlator in the form [9]:

$$
C_{q}=\left|\lambda_{q}\right|^{2}=2 \pi\left\langle\lambda^{2}\right\rangle(2 R)^{2} \mathrm{e}^{-2 q R},
$$

where $C_{q}$ is the Fourier transform of the correlator $C\left(\boldsymbol{r}-\boldsymbol{r}^{\prime}\right), R$ is the spatial scale of fluctuations, and $\boldsymbol{q}=\boldsymbol{k}-\boldsymbol{k}^{\prime}$ is the momentum change due to scattering by fluctuations of the Rashba field.

The charge current operator is defined as $\hat{j}_{i}=e \hat{v}_{i}$, where $\hat{v}_{i}$ is the velocity operator,

$$
\hat{v}_{i}=\frac{\partial H}{\partial k_{i}} .
$$

Thus, the components $\hat{j}_{x(y)}$ can be written explicitly as

$$
\hat{j}_{x(y)}=\frac{e}{m} k_{x(y)} \delta_{\boldsymbol{k} \boldsymbol{k}^{\prime}} \pm \beta e \sigma_{x(y)} \delta_{\boldsymbol{k} \boldsymbol{k}^{\prime}} \mp e \lambda_{\boldsymbol{k} \boldsymbol{k}^{\prime}} \sigma_{y(x)} .
$$

The spin current operator, in turn, is defined as

$$
\hat{j}_{i}^{s_{\alpha}}=\frac{1}{2 e}\left\{\hat{j}_{i}, \hat{s}_{\alpha}\right\}=\frac{1}{4 e}\left\{\hat{j}_{i}, \sigma_{\alpha}\right\},
$$

where $\{\ldots\}$ stands for the anticommutator and $\hat{s}_{\alpha}=$ $\sigma_{\alpha} / 2$. 


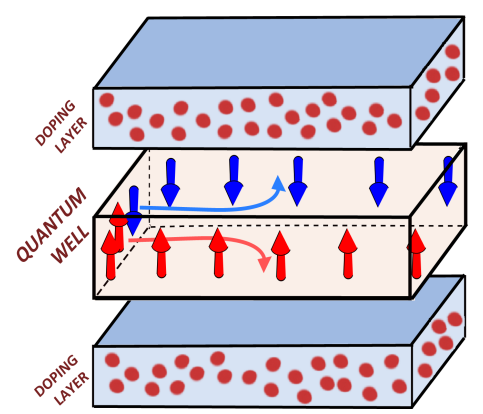

Fig. 1. Schematic picture of spin Hall effect in a quantum well. Due to randomly distributed dopants, there is a random Rashba spin-orbit interaction in the system, in addition to the uniform Dresselhaus one.

To calculate the spin Hall conductivity we use the Kubo formalism. The retarded Green function of the system without perturbation is

$$
G_{\boldsymbol{k}}^{\mathrm{R}}=G_{\boldsymbol{k} 0}^{\mathrm{R}} \sigma_{0}+G_{\boldsymbol{k} x}^{\mathrm{R}} \sigma_{x}+G_{\boldsymbol{k} y}^{\mathrm{R}} \sigma_{y},
$$

where

$$
\begin{aligned}
G_{\boldsymbol{k} 0}^{\mathrm{R}} & =\frac{1}{2}\left(G_{\boldsymbol{k}+}^{\mathrm{R}}+G_{\boldsymbol{k}-}^{\mathrm{R}}\right), \\
G_{\boldsymbol{k} x}^{\mathrm{R}} & =\frac{1}{2} \cos \phi\left(G_{\boldsymbol{k}+}^{\mathrm{R}}-G_{\boldsymbol{k}-}^{\mathrm{R}}\right), \\
G_{\boldsymbol{k} y}^{\mathrm{R}} & =-\frac{1}{2} \sin \phi\left(G_{\boldsymbol{k}+}^{\mathrm{R}}-G_{\boldsymbol{k}-}^{\mathrm{R}}\right),
\end{aligned}
$$

with

$$
G_{\boldsymbol{k} \pm}^{\mathrm{R}}=\frac{1}{\varepsilon-E_{k \pm}+\mathrm{i} \Gamma}
$$

and $\phi$ denoting the angular coordinate of the vector $\boldsymbol{k}$. Here $E_{k \pm}=\varepsilon_{k} \pm \beta k$ are the two electron bands of 2DEG with Dresselhaus interaction, $\varepsilon_{k}=k^{2} / 2 m$, and $\Gamma=1 / 2 \tau$ with $\tau$ denoting the relevant relaxation time. For simplicity, we treat $\Gamma$ as a phenomenological parameter.

\section{Spin Hall conductivity}

Following the Kubo-Streda formula [11], we write the spin Hall conductivity as

$$
\sigma_{x y}^{s_{z}}=\sigma_{x y}^{s_{z} \mathrm{I}}+\sigma_{x y}^{s_{z} \mathrm{II}},
$$

where $\sigma_{x y}^{s_{z} \mathrm{I}}$ is determined by the retarded and advanced Green functions at the Fermi level,

$$
\sigma_{x y}^{s_{z} \mathrm{I}}=\frac{e}{2 \pi} \int \frac{\mathrm{d}^{2} \boldsymbol{k}}{(2 \pi)^{2}} \operatorname{Tr}\left\{\hat{j}_{x}^{s_{z}} G^{\mathrm{R}}\left(\varepsilon_{\mathrm{F}}\right) \hat{v}_{y} G^{\mathrm{A}}\left(\varepsilon_{\mathrm{F}}\right)\right\} .
$$

In turn, $\sigma_{x y}^{s_{z} \mathrm{II}}$ is the contribution to the spin Hall conductivity, which depends on states below the Fermi level. This term, however, vanishes in the system under considerations.

The lowest-order diagrams contributing to the spin Hall conductivity are depicted in Fig. 2. The bare bubble diagram $\left(D_{1}\right)$ corresponds to the intrinsic term. Diagrams $D_{2}$ and $D_{3}$ are connected with the presence of anomalous velocity vertex and they are a consequence of the random Rashba field. Accordingly, the spin Hall conductivity may be written as

$$
\sigma_{x y}^{s_{z}}=\frac{e}{2 \pi} \operatorname{Tr} \sum_{\boldsymbol{k} \boldsymbol{k}^{\prime}}\left(D_{1}+D_{2}+D_{3}\right) .
$$

Since the intrinsic contribution $\sigma_{x y}^{s_{z} \text { int }}$ is given by [12]:

$$
\sigma_{x y}^{s_{z} \text { int }}=\frac{e}{2 \pi} \operatorname{Tr} \sum_{k \boldsymbol{k}^{\prime}} D_{1}=-\frac{e}{8 \pi},
$$

we write $\sigma_{x y}^{s_{z}}$ in the form

$$
\sigma_{x y}^{s_{z}}=-\frac{e}{8 \pi}+\delta \sigma_{x y}^{s_{z}}
$$

The term $\sigma_{x y}^{s_{z}}$ int is universal for the 2DEG when the Dresselhaus spin-orbit interaction is dominant, as already discussed earlier [12].
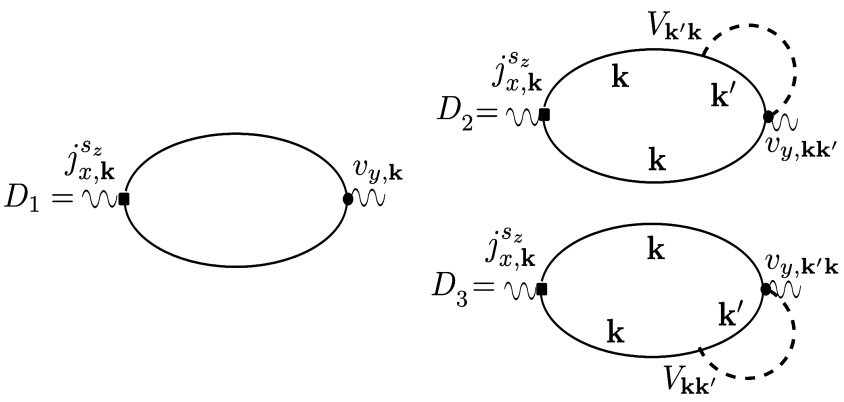

Fig. 2. The Feynman diagrams contributing to the spin Hall conductivity in the dc limit.

Let us consider now the contribution $\delta \sigma_{x y}^{s z}$ due to random Rashba field,

$$
\delta \sigma_{x y}^{s_{z}}=\frac{e}{2 \pi} \operatorname{Tr} \sum_{\boldsymbol{k} \boldsymbol{k}^{\prime}}\left(D_{2}+D_{3}\right) .
$$

Upon calculating contributions from the relevant diagrams one finds

$$
\begin{gathered}
\delta \sigma_{x y}^{s_{z}}=\frac{e}{2 \pi} \sum_{\boldsymbol{k} \boldsymbol{k}^{\prime}} \frac{k_{x}}{2 m}\left(k_{y}+k_{y}^{\prime}\right) \frac{\left|\lambda_{\boldsymbol{k} \boldsymbol{k}^{\prime}}\right|^{2}}{2}\left(T_{1}+T_{3}\right) \\
-\frac{e}{2 \pi} \sum_{\boldsymbol{k} \boldsymbol{k}^{\prime}} \frac{k_{x}}{2 m}\left(k_{x}+k_{x}^{\prime}\right) \frac{\left|\lambda_{\boldsymbol{k} \boldsymbol{k}^{\prime}}\right|^{2}}{2}\left(T_{2}+T_{4}\right),
\end{gathered}
$$

where

$$
\begin{aligned}
T_{1} & +T_{3}=\mathrm{i} \frac{1}{2}\left(\cos \phi \sin \phi^{\prime}+\sin \phi \cos \phi^{\prime}\right) \\
& \times\left(G_{\boldsymbol{k}+}^{\mathrm{A}} G_{\boldsymbol{k}-}^{\mathrm{R}}-G_{\boldsymbol{k}-}^{\mathrm{A}} G_{\boldsymbol{k}+}^{\mathrm{R}}\right) \\
& \times\left(G_{\boldsymbol{k}^{\prime}-}^{\mathrm{A}}-G_{\boldsymbol{k}^{\prime}+}^{\mathrm{A}}+G_{\boldsymbol{k}^{\prime}-}^{\mathrm{R}}-G_{\boldsymbol{k}^{\prime}+}^{\mathrm{R}}\right), \\
T_{2} & +T_{4}=-\mathrm{i} \frac{1}{2}\left(\cos \phi \cos \phi^{\prime}-\sin \phi \sin \phi^{\prime}\right) \\
& \times\left(G_{\boldsymbol{k}+}^{\mathrm{A}} G_{\boldsymbol{k}-}^{\mathrm{R}}-G_{\boldsymbol{k}-}^{\mathrm{A}} G_{\boldsymbol{k}+}^{\mathrm{R}}\right) \\
& \times\left(G_{\boldsymbol{k}^{\prime}+}^{\mathrm{A}}-G_{\boldsymbol{k}^{\prime}-}^{\mathrm{A}}+G_{\boldsymbol{k}^{\prime}+}^{\mathrm{R}}-G_{\boldsymbol{k}^{\prime}-}^{\mathrm{R}}\right) \\
& +\mathrm{i}\left(G_{\boldsymbol{k}+}^{\mathrm{R}} G_{\boldsymbol{k}-}^{\mathrm{A}}+G_{\boldsymbol{k}-}^{\mathrm{R}} G_{\boldsymbol{k}+}^{\mathrm{A}}\right)\left(G_{\boldsymbol{k}^{\prime} 0}^{\mathrm{A}}-G_{\boldsymbol{k}^{\prime} 0}^{\mathrm{R}}\right),
\end{aligned}
$$

with the Green functions taken at the Fermi level.

Taking into account that $\boldsymbol{k}^{\prime}=\boldsymbol{k}-\boldsymbol{q}$, and integrating over $\phi$ one finds

$$
\delta \sigma_{x y}^{s_{z}}=\frac{e \Gamma \sqrt{2}}{16 \pi^{2}} \int \mathrm{d} q q \int \mathrm{d} \theta \int \mathrm{d} k k \frac{C_{q}}{4 m}
$$




$$
\begin{aligned}
& \times \frac{k(2 k-q \cos \theta)}{\left(\varepsilon_{\mathrm{F}}-\varepsilon_{k}\right)^{2}+2 m \beta^{2} \varepsilon_{k}} \\
& \times\left[\left|F_{1}\right| \delta\left(\varepsilon_{k}-\varepsilon_{1}\right)+\left|F_{2}\right| \delta\left(\varepsilon_{k}-\varepsilon_{2}\right)\right] \\
& \times\left[\delta\left(\varepsilon_{\mathrm{F}}-E_{\boldsymbol{k}-\boldsymbol{q}+}\right)+\delta\left(\varepsilon_{\mathrm{F}}-E_{\boldsymbol{k}-\boldsymbol{q}-}\right)\right],
\end{aligned}
$$

where $\theta$ is the angle between vectors $\boldsymbol{k}$ and $\boldsymbol{q}$, while $F_{i}$ $(i=1,2)$ are defined as:

$$
F_{i}=\frac{\left[2 m \beta^{2} \varepsilon_{i}+\left(\varepsilon_{i}-\varepsilon_{\mathrm{F}}\right)^{2}\right]^{3 / 2}}{\left(\varepsilon_{i}-\varepsilon_{\mathrm{F}}\right)^{3}+3 m \beta^{2}\left(\varepsilon_{i}^{2}-\varepsilon_{\mathrm{F}}^{2}\right)-2 m^{2} \beta^{4} \varepsilon_{i}},
$$

with

$$
\varepsilon_{1,2}=m \beta^{2}+\varepsilon_{\mathrm{F}} \mp \sqrt{m^{2} \beta^{4}+2 m \beta^{2} \varepsilon_{\mathrm{F}}} .
$$

The next step is to convert the Dirac delta function $\delta\left(\varepsilon_{\mathrm{F}}-E_{\boldsymbol{k}-\boldsymbol{q} \pm}\right) \Rightarrow \delta\left(\theta-\theta_{n}\right)$, where $\theta_{n}$ are solutions of the equation $\varepsilon_{\mathrm{F}}-E_{\boldsymbol{k}-\boldsymbol{q} \pm}=0$. In Fig. 3 we show the contribution to spin Hall conductivity due to random Rashba field as a function of the Dresselhaus coupling parameter $\beta$. Note that this contribution tends to zero for sufficiently large values of $\beta$.

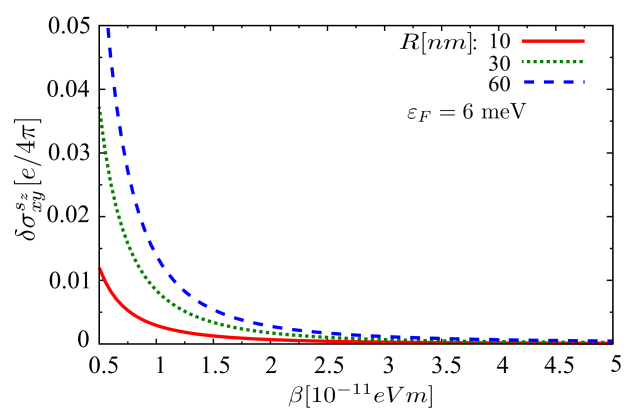

Fig. 3. Contribution $\delta \sigma_{x y}^{s z}$ to the spin Hall conductivity as a function of the Dresselhaus spin-orbit coupling parameter $\beta$ for indicated values of $R$ and $\varepsilon_{\mathrm{F}}$. The other parameters are: $\sqrt{\left\langle\lambda^{2}\right\rangle}=1.5 \times 10^{-12} \mathrm{eV} \mathrm{m}$, $\Gamma=0.5 \mathrm{meV}$, and $m=0.05 \mathrm{~m}_{0}$.

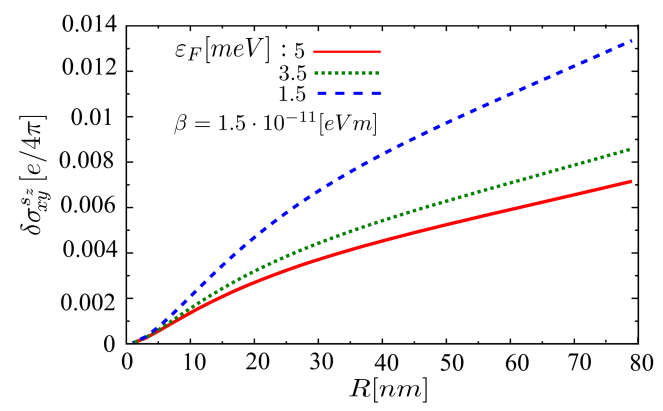

Fig. 4. The contribution $\delta \sigma_{x y}^{s z}$ as a function of the correlation parameter $R$ for indicated values of $\varepsilon_{\mathrm{F}}$ and $\beta$. The other parameters are as in Fig. 3.

In Fig. 4, in turn, the spin Hall conductivity is presented as a function of the parameter $R$ describing cor- relation length of the fluctuating Rashba field. In the limit of long-range correlations $\left(k_{\mathrm{F}} R \gg 1\right)$ the spin Hall conductivity is a linear function of $R$, but for $k_{\mathrm{F}} R \ll 1$ the term $\delta \sigma_{x y}^{s z}$ is suppressed and tends to zero as a function of $R^{2}$. This result is consistent with previous results for system with random Rashba spin-orbit interaction only [10].

\section{Conclusion}

We have analyzed SHE in a two-dimensional electron gas with constant Dresselhaus spin-orbit coupling and fluctuating Rashba interaction. In such a case, the spin Hall conductivity has a universal intrinsic contribution, $\sigma_{x y}^{s_{z} \text { int }}$, and an additional term, $\delta \sigma_{x y}^{s_{z}}$. The latter contribution, however, is suppressed for sufficiently strong Dresselhaus spin-orbit interaction.

\section{Acknowledgments}

This work has been supported by the European Union under European Social Fund - Operational Programme Human Capital — POKL.04.01.01-00-133/09-00 and by National Center of Science (NCN, Poland) as a research project No. DEC-2011/03/N/ST3/02353 for years 20122014. The authors also acknowledge valuable discussions with V.K. Dugaev.

\section{References}

[1] M.I. Dyakonov, V.I. Perel, Pis'ma Zh. Eksp. Teor. Fiz. 13, 657 (1971); JETP Lett. 13, 467 (1971).

[2] M.I. Dyakonov, V.I. Perel, Phys. Lett. A 35, 459 (1971).

[3] J.E. Hirsch, Phys. Rev. Lett. 83, 1834 (1999).

[4] J. Schliemann, Int. J. Mod. Phys. B 20, 1015 (2006).

[5] A. Engel, E.I. Rashba, B.I. Halperin, in: Handbook of Magnetism and Advanced Magnetic Materials, Eds. H. Kronmuller, S. Parkin, Vol. 5, Wiley, New York 2007, p. 1.

[6] M.I. Dyakonov, A.V. Khaetskii, in: Spin Physics in Semiconductors, Ed. M.I. Dyakonov, Springer-Verlag, Berlin 2008, Chap. 8.

[7] G. Vignale, J. Supercond. Nov. Magn. 23, 3 (2010).

[8] J. Sinova, D. Culcer, Q. Niu, N.A. Synitsyn, T. Jungwirth, A.H. MacDonald, Phys. Rev. Lett. 92, 126603 (2004).

[9] M.M. Glazov, E.Ya. Sherman, V.K. Dugaev, Physica E 42, 2157 (2010).

[10] V.K. Dugaev, M. Inglot, E.Ya. Sherman, J. Barnaś, Phys. Rev. B 82, 121310(R) (2010).

[11] P. Streda, J. Phys. C, Solid State Phys. 15, L717 (1982).

[12] N.A. Sinitsyn, E.M. Hankiewicz, W. Teizer, J. Sinova, Phys. Rev. B 70, 081312(R) (2004). 